\title{
Effects of Different External Loads on the Activities of the Gluteus Maximus and Biceps Femoris during Prone Hip Extension in Healthy Young Men
}

\author{
Chang-Hwan Bae, PT, PhD • Yu-Won Choe, PT, MS ${ }^{1} \cdot$ Myoung-Kwon Kim, PT, $\mathrm{PhD}^{2 \dagger}$
}

Bae Chang Hwan Training Center, Samsan-ro, Nam-gu, Ulsan, 37, Republic of Korea

${ }^{1}$ Department of Rehabilitation Sciences, Graduate School, Daegu University

${ }^{2}$ Department of Physical Therapy, College of Rehabilitation Sciences, Daegu University

Received: December 30, 2019 / Revised: March 10, 2020 / Accepted: March 7, 2020

(C) 2020 J Korean Soc Phys Med

\section{| Abstract |}

PURPOSE: This study examined the effects of different external loads on the muscle activities around the hip during prone hip extension with knee flexion (PHEKF) exercise in healthy young men.

METHODS: Sixteen healthy adult males participated in the study. A pressure biofeedback unit was used to provide feedback to the participants during the abdominal drawing-in maneuver (ADIM) with PHEKF. Sandbags ( $0 \mathrm{~kg}, 1 \mathrm{~kg}, 2 \mathrm{~kg}$, and $3 \mathrm{~kg}$ ) were used to provide external resistance. The quadriceps was contracted to maintain knee flexion $90^{\circ}$ against resistance. Each resistance condition using a sandbag weight was given in random order. Surface electromyography (sEMG) was used to measure the electrical activity of the gluteus maximus, biceps femoris, and erector spinae during PHEKF.

RESULTS: The muscle activity of the gluteus maximus

†Corresponding Author : Myoung-Kwon Kim

skybird-98@hanmail.net, https://orcid.org/0000-0002-7251-6108 This is an Open Access article distributed under the terms of the Creative Commons Attribution Non-Commercial License (http://creativecommons.org/licenses/by-nc/3.0) which permits unrestricted non-commercial use, distribution, and reproduction in any medium, provided the original work is properly cited. was highest with the $3 \mathrm{~kg}$ resistance and lowest with $0 \mathrm{~kg}$ (F $=128.46, \mathrm{P}=.00)$. The muscle activities of the biceps femoris and erector spinae were highest with $0 \mathrm{~kg}$ and lowest with 3 $\mathrm{kg}(\mathrm{F}=29.49, \mathrm{P}=.00)$. The muscle activity rate of the gluteus maximus/biceps femoris was highest with $3 \mathrm{~kg}$ and lowest with $0 \mathrm{~kg}(\mathrm{~F}=37.49, \mathrm{P}=.00)$.

CONCLUSION: The activity of the gluteus maximus was increased using a higher external weight load during PHEKF, while the activity of the biceps femoris decreased. These findings suggest that an external weight is needed during hip extensor exercise to strengthen the gluteus maximus and inhibit the biceps femoris.

Key Words: Electromyography, External loads, Gluteus maximus, Prone hip extension

\section{Introduction}

Hip extension exercise is the general clinical method for improving the weakened muscle strength of the hip extensors [1,2]. Hip extension exercise is often used to strengthen the gluteus maximus, and is also recommended for patients with pain in the lower back, pelvis, or hip [2-4]. The gluteus maximus contributes to the postural 
alignments, core stability, and functional abilities that are important for normal gait [5]. A weakness of gluteus maximus can cause an abnormal gait cycle that can affect the gait mechanics at both hip and knee joints [5-7]. In particular, gluteus maximus exercise is important for patients who have low back pain and lower extremity injuries [8]. Patients with low back pain show reduced activity of the gluteus maximus [3,9-11]. Early activation of the hamstring muscles and spinal erector, as well as delayed or decreased activation of the gluteal muscles, are considered indications of faulty muscle activation $[1,12,13]$.

Many studies have assessed methods for selective strengthening of the gluteus maximus. Prone hip extension (PHE) exercise is generally used to activate and strengthen the gluteus maximus selectively $[14,15]$. Active prone hip extension with knee flexion is a common exercise employed to optimize gluteus maximus activation because this position causes active insufficiency of the hamstring [11]. A previous study reported that PHE exercise is the most effective method for activating the gluteal muscles when subjects extend their hip with $30^{\circ}$ abduction [8].

Other studies have suggested that an abdominal drawing-in maneuver (ADIM) with prone hip extension is useful for the selective activation of the gluteus maximus from the erector spinae and hamstring $[2,16]$. The hamstring activation earlier than the gluteal muscles contributes to pain and hip dysfunction $[1,13,17]$. A correction of the abnormal pattern is recommended to reduce the stress on the spine and hip joint $[1,12,13]$. In a clinical setting, the ADIM is used during hip extension to prevent abnormal motion of the pelvis and lumbar [16].

Previous studies have shown that the activity of the gluteus maximus is related to the hamstring activity $[3,13,18]$, and the degree of knee extension has an effect on the gluteus maximus activity $[11,19]$. When the level of knee extension is increased, the activity of the gluteus maximus decreases, and the hamstring activity increases $[11,19]$. Previous studies compared the muscle activities of the hamstring and gluteus maximus during prone hip extension with ADIM [20-22]. The following were applied during active prone hip extension exercise: knee flexion, knee extension, hip external rotation with knee flexion, and hip external rotation with knee extension [21]. In addition, eleven different exercises of the gluteus maximus used in therapeutic exercises were compared [22]. On the other hand, few studies have examined the effects of the external weight loads on the muscle activities around hip during prone hip extension for selective strengthening of the gluteus maximus. Therefore, this study examined the effects of an external weight load on the muscle activities around the hip during prone hip extension with knee flexion (PHEKF) exercise.

\section{Methods}

\section{Participants}

Sixteen healthy adult males in their 20 s, who agreed voluntarily to this experiment, were included. The sample size for this study was calculated using the $\mathrm{G}^{*}$ Power program 3.1.0 (G power program Version 3.1, HeinrichHeine-University Düsseldorf, Germany). Based on the data from a pilot study, the estimated sample size required to obtain a minimum power of $80 \%$ at a significant alpha level of $95 \%$ was 14 . Accordingly, 16 participants were recruited to account for a possible dropout rate of $20 \%$.

The participants had an average age, height, weight, and BMI of $20.60 \pm 3.33$ years, $176.93 \pm 4.69 \mathrm{~cm}, 74.20$ $\pm 8.17 \mathrm{~kg}$, and $23.71 \pm 1.90 \mathrm{~kg} / \mathrm{m}$, respectively (Table 1).

Table 1. General Characteristics of the Participants

\begin{tabular}{ccc}
\hline General Characteristics & Mean \pm SD & Range \\
\hline Sex (male/female) & $16 / 0$ & \\
Age (years) & $20.60 \pm 3.33$ & $23 \sim 29$ \\
Height $(\mathrm{cm})$ & $176.93 \pm 4.69$ & $169 \sim 186$ \\
Weight $(\mathrm{kg})$ & $74.20 \pm 8.17$ & $65 \sim 89$ \\
BMI $\left(\mathrm{kg} / \mathrm{m}^{2}\right)$ & $23.71 \pm 1.90$ & $20.98 \sim 26.42$ \\
\hline
\end{tabular}

Mean $\pm \mathrm{SD},{ }^{*} \mathrm{p}<.05$ 


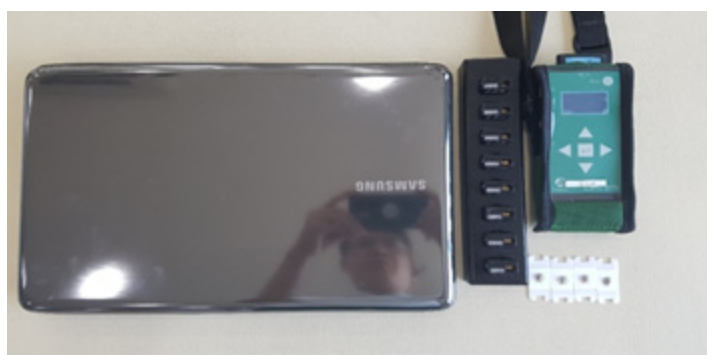

Fig. 1. Noraxon myosystem DTS.

Before starting the study, all subjects understood its content and signed an informed consent form. This study was approved by the ethical committee of Daegu University (1040621-201911-HR-027-02) and complied with the ethical standards of the declaration of Helsinki. The exclusion criteria for the participants included the following: (1) a history of hip or back pain lasting for more than one week within the last five years before testing [13], (2) current lower extremity injury [13], (3) lumbar or hip pain when performing prone hip extension with knee flexion [8], (4) Thomas test at $10^{\circ}$ below the hip extension and knee flexion below $80^{\circ}$ [3], and (5) hip pain with active straight-leg raises or passive hip flexion with adduction and medial rotation [13].

\section{Measurements}

\section{1) Surface Electromyography (sEMG)}

The electrical activity of the muscles was measured by sEMG (Noraxon myosystem DTS, USA) (Fig. 1). The sEMG data were recorded, and the muscle activities of the gluteus maximus, biceps femoris, and erector spinae on the dominant side were analyzed. $\mathrm{Ag} / \mathrm{AgC}$ surface electrodes were used for the sEMG measurements. The electrodes were disposable grounded electrodes with an electrode area of $4.7 \mathrm{~cm}^{2}$. The inter-electrode distance was attached to the surface parallel to the muscle fibers to 2 $\mathrm{cm}$ [23]. Before measuring sEMG, any hair on the skin was shaved, which was then cleaned with an alcohol swab.

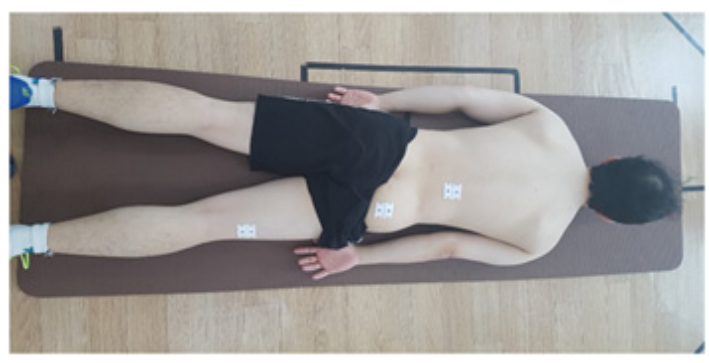

Fig. 2. Surface electromyography.

All electrodes were attached to the muscles based on anatomical landmarks [24](Fig. 2) The gluteus maximus electrodes were attached half the distance between the greater trochanter and second sacral vertebra and at an oblique angle, or slightly above, the level of the trochanter [24]. Biceps femoris electrodes were attached a percentage distance from the ischial tuberosity to the lateral side of the popliteus cavity [24]. The erector spinae electrodes were attached approximately $2 \mathrm{~cm}$ lateral to the spinous process at the L1 level and aligned parallel to the spine [24].

The electromyography (EMG) data were collected using a TeleMyo DTS (Noraxon myosystem DTS, USA). A sampling rate of $1,500 \mathrm{~Hz}$ was used for the raw EMG signal acquisition, and the signals were full-wave rectified. Band-pass filtering at $30-500 \mathrm{~Hz}$ was performed using the MyoResearch XP 1.72 (Noraxon Inc., Holtsville, NY, USA) software, and the signals were also notch filtered at $60 \mathrm{~Hz}$ to remove noise. To normalize the EMG data, a maximum voluntary isometric contraction (MVIC) was performed for each muscle, and the EMG amplitude was recorded. The mean of the EMG data between 2-4 seconds was processed using the root-mean-square (RMS) and represent $100 \%$ MVIC. For the MVIC of the gluteus maximus, the participants were asked to maintain hip extension with $90^{\circ}$ knee flexion against a resistance in the prone position [25]. The MVIC of the biceps femoris was performed in the prone position, with the knee flexed with a resistance applied just above the ankle [26,27]. For the MVIC of the erector spinae, the participants were asked 


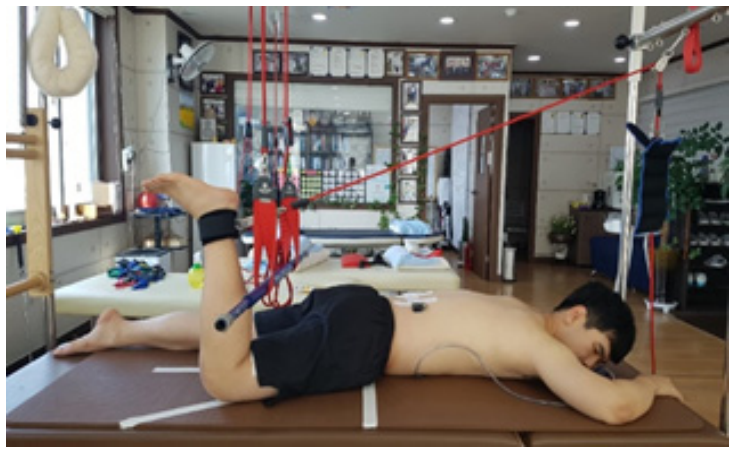

Starting position

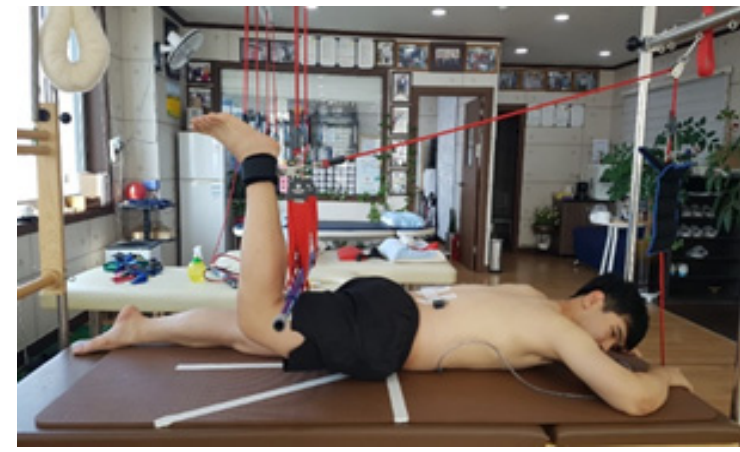

Ending position

Fig. 3. Prone hip extension with knee flexion.

to raise their trunk against the resistance in the prone position. At the same time, the lower extremities were stabilized firmly on the table [28]. A five-minute break was allowed between each test to prevent muscle fatigue [24].

\section{Experimental Procedures}

\section{1) Abdominal Drawing-in Maneuver (ADIM)}

The participants received education about ADIM and exercise method using a pressure biofeedback unit (PBFU, Stabilizer, Chattanooga, USA) for ten minutes before the experiment began. The participants laid down in the prone position, and the PBFU was placed between the table and the participants' lower abdomen [29]. The participants performed the ADIM in the prone position using a PBFU to control the pelvic posterior tilt [16]. The participants were then asked to draw in the abdomen and hold this position, so the change in pressure could be measured [29]. The PBFU was inflated until the pressure reached 70 $\mathrm{mmHg}$, and the participant and investigator monitored this pressure during the ADIM to ensure that it remained at $60 \mathrm{mmHg}$ [16]. Data collected within pressure changes of $\pm 5 \mathrm{mmHg}$ were used for statistical analysis [16].

2) Prone Hip Extension with Knee Flexion (PHEKF) PHEKF was used for selective strengthening of the gluteus maximus while inhibiting the activity of the biceps femoris [14]. The participants performed PHEKF in $90^{\circ}$ knee flexion with $30^{\circ}$ hip abduction and $10^{\circ}$ hip extension to increase the muscle activity of gluteus maximus [8]. After measuring the degree of prone hip extension using a goniometer (Prstige medical, USA), a horizontal bar was hung by a rope and used as a reference point when the participants reached $10^{\circ}$ hip extension (Fig. 3). In the prone position, the participant performed $10^{\circ}$ hip extension until the posterior knee reached the horizontal bar [30]. The participants maintained $90^{\circ}$ knee flexion with $30^{\circ}$ hip abduction and $10^{\circ}$ hip extension in the prone position against the resistance. Sandbags $(0 \mathrm{~kg}, 1 \mathrm{~kg}, 2 \mathrm{~kg}$, and $3 \mathrm{~kg}$ ) were used to provide the resistance on the ankle through a rope and pulley. The quadriceps muscles were contracted to maintain $90^{\circ}$ knee flexion against a knee flexion force. Each resistance condition using a sandbag weight was given in random order. Each condition was tested three times for five seconds each. A five-minute break was allowed between the tests.

\section{Statistical Analysis}

The data were analyzed using SPSS version 18.0 (SPSS Inc. Chicago, IL) for Windows. The changes in the muscle activity of gluteus maximus, biceps femoris, and erector spinae associated with four resistance conditions during 
Table 2. Muscle Activity Around Hip Associated with External Weight Loads

\begin{tabular}{cccccccc}
\hline \multirow{2}{*}{ Muscles } & \multicolumn{9}{c}{ Weight Loads } & \multirow{2}{*}{$\mathrm{F}$} & $\mathrm{p}$ \\
\cline { 2 - 5 } & $0 \mathrm{~kg}$ & $1 \mathrm{~kg}$ & $2 \mathrm{~kg}$ & $3 \mathrm{~kg}$ & & \\
\hline GM & $37.50 \pm 3.31$ & $42.18 \pm 4.10$ & $45.06 \pm 4.07$ & $49.06 \pm 4.11$ & 128.469 & .000 * \\
BF & $20.85 \pm 4.02$ & $14.12 \pm 2.85$ & $10.12 \pm 1.84$ & $8.12 \pm 1.49$ & 29.493 & .000 * \\
ES & $32.00 \pm 3.59$ & $30.81 \pm 3.59$ & $27.81 \pm 3.33$ & $27.37 \pm 4.01$ & 73.851 & .000 * \\
GM/BF & $2.14 \pm 1.28$ & $4.61 \pm 3.82$ & $6.38 \pm 3.81$ & $9.67 \pm 6.84$ & 37.498 & .000 * \\
\hline
\end{tabular}

Mean $\pm \mathrm{SD},{ }^{*} \mathrm{p}<.05$

GM: Gluteus Maximus, BF: Biceps Femoris, ES: Erector Spinae
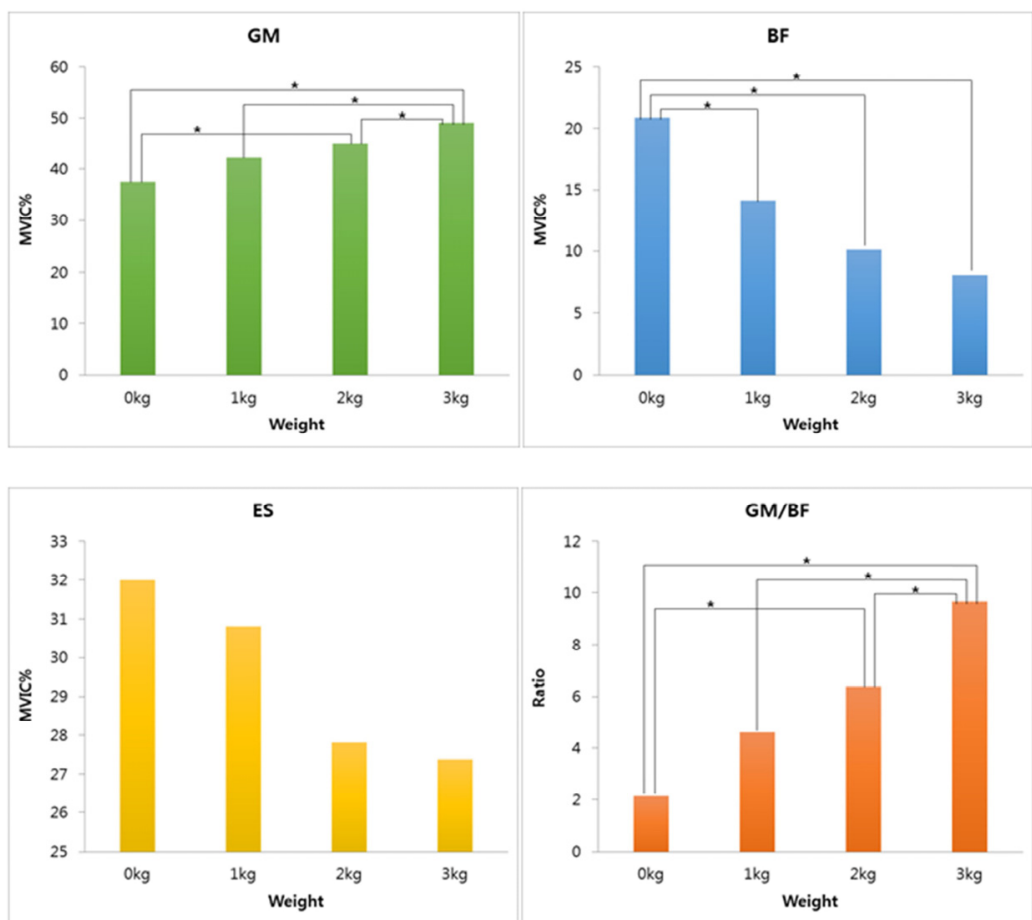

Fig. 4. Post-hoc test of the muscle activity around hip associated with external weight loads.

the PHEKF were determined using one-way repeated ANOVA. A Bonferroni correction was used for the post-hoc test. The significance levels were set to $\alpha=.05$.

\section{Results}

The activity of the gluteus maximus and the muscle activity rate of the gluteus maximus/biceps femoris were highest with $3 \mathrm{~kg}$ resistance and lowest with $0 \mathrm{~kg}$ (Table 2). Repeated measure analysis revealed significant differences in muscle activities associated with the changes in external weight loads $(p<.05)$. A comparison of the resistance revealed significant differences between $0 \mathrm{~kg}$ and $2 \mathrm{~kg}$, $0 \mathrm{~kg}$ and $3 \mathrm{~kg}, 1$ and $3 \mathrm{~kg}$, and 2 and $3 \mathrm{~kg}(\mathrm{p}<.008)$ (Fig. 4).

The activities of the biceps femoris and erector spinae were highest with $0 \mathrm{~kg}$ resistance and lowest with $3 \mathrm{~kg}$ 
(Table 2). Repeated measure analysis revealed significant differences in the muscle activities associated with the changes in the external weight loads $(p<.05)$. A comparison of the resistances revealed significant differences between 0 $\mathrm{kg}$ and $1 \mathrm{~kg}, 0 \mathrm{~kg}$ and $2 \mathrm{~kg}$, and $0 \mathrm{~kg}$ and $3 \mathrm{~kg}(\mathrm{p}<$ .008)(Fig. 4) in the biceps femoris, but there were no significant differences between the resistances $(\mathrm{p}<$ .008)(Fig. 4) in the erector spinae.

\section{Discussion}

This study examined the effects of external weight loads with PHEKF and ADIM on the muscle activities around the hip. The activity of the gluteus maximus increased with increasing external weight load during PHEKF while the activity of the biceps femoris decreased.

During hip extension, when the hamstring is taut, or gluteus maximus activation is delayed, the hamstring is activated more than the gluteus maximus. Therefore, this study used PHEKE in $90^{\circ}$ knee flexion with $30^{\circ}$ hip abduction to strengthen the gluteus maximus selectively and inhibit hamstring activation [31].

The muscle activity of the gluteus maximus was highest with $3 \mathrm{~kg}$ external loads and lowest with $0 \mathrm{~kg}$. Excessive knee flexion has been considered a sign of hamstring over-activity $[13,32,33]$. In this study, weights to provide the quadriceps with resistance play a role in preventing hamstring activation. Neurophysiologically, the hamstring activity decreases during quadriceps contraction [34]. The gluteus maximus activity is low while the hamstring is activated $[1,12,13]$, and the greatest activity of the gluteus maximus is related to the lowest activity for the hamstring [11]. Since the quadriceps had to contract to maintain knee flexion $90^{\circ}$ against external weight loads given by the weights, the quadriceps activity increased with increasing external load. Therefore, when a larger external load was given, the hamstring showed less activation with a concomitant increase in gluteus maximus activation. In this study, the muscle activity of the gluteus maximus was highest with $3 \mathrm{~kg}$ resistance during PHEKF with ADIM. Under the condition of $0 \mathrm{~kg}$ resistance, the biceps femoris was activated more than that under the $1 \mathrm{~kg}, 2 \mathrm{~kg}$, and $3 \mathrm{~kg}$ conditions. As a result of increasing biceps femoris activity, the muscle activity of gluteus maximus was lowest at $0 \mathrm{~kg}$ resistance. A higher external load was found to be related to a higher activity of the gluteus maximus and lower activity biceps femoris.

The muscle activity of the biceps femoris was highest with a $0 \mathrm{~kg}$ external load and lowest with a $3 \mathrm{~kg}$ load. The hamstring activity is related to the activity of the gluteus maximus [1,11-13]. The hamstring showed the highest EMG activity, and the gluteus maximus showed the lowest activity. The greatest activity of the gluteus maximus is related to the lowest activity of the hamstring [11]. The hamstring activity is also affected by the degree of knee flexion [35,36]. A previous study showed that the hamstring activity was lowest during hip extension with knee flexion [11]. When the quadriceps muscle was activated to maintain $90^{\circ}$ knee flexion, the hamstring activity was relatively low. In this study, the muscle activity of the biceps femoris was lowest with $3 \mathrm{~kg}$ resistance during PHEKF with ADIM. With $0 \mathrm{~kg}$ resistance, the biceps femoris was activated more than under the $1 \mathrm{~kg}, 2 \mathrm{~kg}$, and $3 \mathrm{~kg}$ loads. The muscle activity of biceps femoris was low because of the increasing knee extensor activity to maintain the knee degree.

The muscle activity of the erector spinae was highest with a $0 \mathrm{~kg}$ external load and lowest with a $3 \mathrm{~kg}$ load. A weak activation or delayed recruitment of the gluteus maximus leads to compensatory stress on the lumbar with over-activity of the erector spinae [17]. In this study, the activity of the erector spinae tended to increase with decreasing activity of the gluteus maximus. These findings are consistent with previous studies suggesting that the increased activity of the erector spinae is associated with a low activity of the gluteus maximus [17]. Although the 
participants performed ADIM to stabilize the lumbar motion and prevent activation of the erector spinae, the erector spinae activity increased in the low resistance condition. Under the $0 \mathrm{~kg}$ condition, biceps femoris activity increased with decreasing quadriceps activity, and the erector spinae activity increased with decreasing activity of the gluteus maximus. As the quadriceps, hamstrings, gluteus maximus, rectus abdominis, and erector spinae are linked functionally and kinetically [5], as the activity of one muscle was increased, the activity of the other muscle was decreased.

No significant differences were observed with increasing external loads in erector spinae. This is because knee extension resistance caused quadriceps contraction and hamstring relaxation, which increased the activation of the gluteus maximus with hamstring inhibition during hip extension. During ADIM exercise, knee extension resistance could not change erector spinae activation because the pelvic posterior tilt changed the length of the erector spinae.

The muscle activity rate of the gluteus maximus/biceps femoris was highest with a $3 \mathrm{~kg}$ external load and the lowest with $0 \mathrm{~kg}$. These results mean that the muscle activity of the gluteus maximus increased while the biceps femoris activity decreased. These results are consistent with previous studies. Previous studies suggested that the gluteus maximus activity is related to the hamstring activity [1,11-13]. In addition, the highest activity of the gluteus maximus is associated with the lowest activity of the hamstring [11], and the gluteus maximus activity is low when the hamstring is activated $[1,12,13]$. Overall, the increased activity of the gluteus maximus was associated with the decreased activities of the biceps femoris and erector spinae. Compared to low external loads, high external loads help activate the gluteus maximus selectively.

This study had several limitations. First, the small sample size might have influenced certain variables and the results. Second, this study was aimed at males who were in good health. Hence, the effects of gender were not assessed. Third, the long-term effects of external loads with PHEKF and ADIM for gluteus maximus strengthening were not examined. Fourth, there is the possibility of a measurement error of EMG. Finally, this study measured the EMG activities of the gluteus maximus, biceps femoris, and erector spinae, but this is insufficient to identify the interactions between the muscles around the trunk, pelvic, hip, and lower extremities. Therefore, further studies will be needed to find the long-term effects, difference between genders, and activities of more muscles.

\section{Conclusion}

This study suggests a method for strengthening exercise of the gluteus maximus to prevent low back pain and injury. In this study, the results showed that the activity of the gluteus maximus increased with a higher external weight load during PHEKF while the activity of the biceps femoris decreased. These findings suggest that external weight loads are needed during hip extensor exercise to strengthen the gluteus maximus and inhibit the biceps femoris.

\section{Acknowledgment}

This material is based on work supported by the Ministry of Trade, Industry \& Energy (MOTIE, Korea) under the Industrial Technology Innovation Program. No.20001123, 'Advanced design development of the design of integrated sleep optimization platform service through core stretching and motion air cell mattress based on pressure sensors and IoT technology'

\section{References}

[1] Sahrmann SA. Diagnosis and treatment of movement impairment syndromes. St. Louis. Mosby Inc. 2002.

[2] Kim TW, Kim YW. Effects of abdominal drawing-in during prone hip extension on the muscle activities of 
the hamstring, gluteus maximus, and lumbar erector spinae in subjects with lumbar hyperlordosis. J Phys Ther Sci. 2015;27(2):383-6.

[3] Vogt L, Banzer W. Dynamic testing of the motor stereotype in prone hip extension from neutral position. Clin Biomech. 1997;12(2);122-7.

[4] Tateuchi H, Taniguchi M, Mori N, et al. Balance of hip and trunk muscle activity is associated with increased anterior pelvic tilt during prone hip extension. J Electromyogr Kinesiol. 2012;22(3):391-7.

[5] Khaiyat OA, Norris J. Electromyographic activity of selected trunk, core, and thigh muscles in commonly used exercises for ACL rehabilitation. J Phys Ther Sci. 2018;30(4):642-8.

[6] Dingenen B, Janssens L, Claes S, et al. Lower extremity muscle activation onset times during the transition from double-leg stance to single-leg stance in anterior cruciate ligament reconstructed subjects. Clin Biomech. 2016; 35:116-23.

[7] Huxel Bliven KC, Anderson BE. Core stability training for injury prevention. Sports health. 2013;5(6):514-22.

[8] Kang SY, Jeon HS, Kwon O, et al. Activation of the gluteus maximus and hamstring muscles during prone hip extension with knee flexion in three hip abduction positions. Man Ther. 2013;18(4):303-7.

[9] Leinonen V, Kankaanpää M, Airaksinen O, et al. Back and hip extensor activities during trunk flexion/extension: effects of low back pain and rehabilitation. Arch Phys Med Rehabil. 2000;81(1):32-7.

[10] Kankaanpää M, Taimela S, Laaksonen D, et al. Back and hip extensor fatigability in chronic low back pain patients and controls. Arch Phys Med Rehabil. 1998; 79(4):412-7.

[11] Sakamoto ACL, Teixeira-Salmela LF, Rodrigues DPF, et al. Gluteus maximus and semitendinosus activation during active prone hip extension exercises. Braz J Phys Ther. 2009;13(4):335-42.

[12] Janda V, Frank C, Liebenson C. Evaluation of muscular imbalance. Rehabilitation of the spine: a practitioner's manual. Baltimore. Lippincott Williams \& Wilkins. 1996.

[13] Lewis CL, Sahrmann SA. Muscle activation and movement patterns during prone hip extension exercise in women. J Athl Train. 2009;44(3):238-48.

[14] Distefano LJ, Blackburn JT, Marshall SW, et al. Gluteal muscle activation during common therapeutic exercises. J Orthop Sports Phys Ther. 2009;39(7):532-40.

[15] Wilson J, Ferris E, Heckler A, et al. A structured review of the role of gluteus maximus in rehabilitation. New Zealand Journal of Physiotherapy. 2005;33(3):95-100.

[16] Oh JS, Cynn HS, Won JH, et al. Effects of performing an abdominal drawing-in maneuver during prone hip extension exercises on hip and back extensor muscle activity and amount of anterior pelvic tilt. J Orthop Sports Phys Ther. 2007;37(6):320-4.

[17] Frank C, Page P, Lardner R. Assessment and treatment of muscle imbalance: the Janda approach. Human kinetics. 2009.

[18] Lehman GJ, Lennon D, Tresidder B, et al. Muscle recruitment patterns during the prone leg extension. BMC Musculoskelet Disord. 2004;5(1):3.

[19] Tassi N, Engrácia VV. Electromyographyc evaluation of movements of lower limb in double pulley system equipment: comparison between gastrocnemius (caput laterale) and gluteus maximus. Electromyogr Clin Neurophysiol. 2007;47(6):293-9.

[20] Oh JS, Cynn HS, Won JH, et al. Effects of performing an abdominal drawing-in maneuver during prone hip extension exercises on hip and back extensor muscle activity and amount of anterior pelvic tilt. J Orthop Sports Phys Ther. 2007;37(6):320-4.

[21] Sakamoto AC, Teixeira-Salmela LF, de Paula-Goulart FR, et al. Muscular activation patterns during active prone hip extension exercises J Electromyogr Kinesiol. 2009; 19(1):335-42.

[22] Selkowitz DM, Beneck GJ, Powers CM. Comparison of electromyographic activity of the superior and inferior 
portions of the gluteus maximus muscle during common therapeutic exercises. J Orthop Sports Phys Ther. 2016; 46(9):794-9.

[23] Cram JR, Kasman GS, Holtz J. Introduction to surface electromyography (1st ed). Aspen Publisher Incorporation. Gaithers-burg. 1998.

[24] Criswell E. Cram's introduction to surface electromyography. Jones \& Bartlett Publishers. 2010.

[25] Willcox EL, Burden AM. The influence of varying hip angle and pelvis position on muscle recruitment patterns of the hip abductor muscles during the clam exercise. J Orthop Sports Phys Ther. 2013;43(5):325-31.

[26] Montgomery HJHJ, Avers D. Daniels and Worthingham's Muscle Testing: Techniques of Manual Examination. Saunders. 1996.

[27] Chan MK, Chow KW, Lai AY, et al. The effects of therapeutic hip exercise with abdominal core activation on recruitment of the hip muscles. BMC Musculoskelet Disord. 2017;18(1):313.

[28] Ansari B, Bhati P, Singla D, et al. Lumbar Muscle Activation Pattern During Forward and Backward Walking in Participants With and Without Chronic Low Back Pain: An Electromyographic Study. J Chiropr Med. 2018;17(4):217-25.

[29] Kim CY, Lee JS, Kim HD, et al. Short-term effects of respiratory muscle training combined with the abdominal drawing-in maneuver on the decreased pulmonary function of individuals with chronic spinal cord injury: A pilot randomized controlled trial. J Spinal Cord Med. 2017;40(1):17-25.

[30] Noh KH, Moon SN, Lee DK, et al. Influence of internal and external stabilization methods during prone hip extension on the selective activation of the gluteus maximus. Phys Ther Korea. 2011;18(3):1-7.

[31] Kang SY, Jeon HS, Kwon O, et al. Activation of the gluteus maximus and hamstring muscles during prone hip extension with knee flexion in three hip abduction positions. Man Ther. 2013;18(4):303-7.

[32] Prentice WE, Voight ML. Techniques in musculoskeletal rehabilitation. McGraw Hill Professional. 2001.

[33] Hertling D, Kessler RM. Management of common musculoskeletal disorders: physical therapy principles and methods (4th ed). Baltimore. Lippincott Williams \& Wilkins. 2006.

[34] Lundy-Ekman L. Neuroscience: Fundamentals for Rehabilitation (3rd ed). St. Louis, MO: Sunders Elsevier. 2007.

[35] Read PJ, Turner AN, Clarke R, et al. Knee angle affects posterior chain muscle activation during an isometric test used in soccer players. Sports. 2019;7(1):13.

[36] Monajati A, Larumbe-Zabala E, Goss-Sampson M, et al. Analysis of the hamstring muscle activation during two injury prevention exercises. J Hum Kinet. 2017; 60(1):29-37. 